\title{
Embracing Technology for Capacity Building in Mental Health: New Path, Newer Challenges
}

\author{
Barikar C. Malathesh ${ }^{1}$ • Ferose Aziz Ibrahim ${ }^{1}$ • P. Lakshmi Nirisha ${ }^{1}$ • \\ Channaveerachari Naveen Kumar ${ }^{1}$ - Prabhat Kumar Chand ${ }^{1} \cdot$ Narayana Manjunatha $^{1}$. \\ Suresh Bada Math ${ }^{1}$ • Jagadisha Thirthalli ${ }^{1}$ • Adarsha Alur Manjappa ${ }^{2}$. \\ Rajani Parthasarathy ${ }^{2} \cdot$ Shanivaram Reddy $^{3} \cdot$ Sanjeev Arora ${ }^{4}$
}

Accepted: 15 November 2020/ Published online: 19 November 2020

(C) Springer Science+Business Media, LLC, part of Springer Nature 2020

\begin{abstract}
Technology driven capacity building initiatives are the way to break the barrier of shortage of mental health human resources in India. This new path, while is a welcome step, comes with its own set of challenges. In one prototypic project that is being implemented in Ramanagara District of Karnataka, a south Indian state, we encountered many such issues. They ranged from issues related to availability of dedicated space to set up the hub-studio, inconsistent internet connectivity (particularly in remote areas) and inadequate digital literacy among the grassroot community health workers who hail from villages. This article summarises these challenges and ends by looking into ways and means of overcoming them.
\end{abstract}

Keywords Telementoring $\cdot \mathrm{ICMR} \cdot \mathrm{ECHO} \cdot \mathrm{CHW}$ 's

\section{Background}

An acute shortage of trained mental health professionals is one of the many reasons for the burgeoning treatment gap in mental illnesses. The use of technology is proposed as a solution

Channaveerachari Naveen Kumar nkumar@nimhans.ac.in

1 Department of Psychiatry, Community Psychiatry Unit, National Institute of Mental Health and Neurosciences (NIMHANS), Bengaluru, Karnataka 560029, India

2 Department of Health and Family Welfare, Government of Karnataka, India

3 Department of Psychiatric Social Work, National Institute of Mental Health and Neurosciences (NIMHANS), Bengaluru, Karnataka 560029, India

4 The ECHO Institute, University of New Mexico, Albuquerque, NM, USA 
[1]. Multiple such technology-driven capacity building initiatives are underway in India that have shown promise and have contributed to bridging the treatment gap [2-8].

All these efforts are conceptually speaking connecting a hub (manned by a specialist in mental health areas) with distant spokes consisting of non-specialist healthcare workforce (including MBBS doctors, AYUSH doctors, Assistant Medical Officers, counsellors, psychologists, social workers, nurses, ASHAs, etc). While these technology-driven innovations appear promising, certain challenges have started to emerge, particularly when these are applied to non-metro areas. This report summaries these impediments and provides future recommendations. To illustrate our case we have carefully examined one prototype digitally-enabled capacity building project at the National Institute of Mental Health and Neurosciences (NIMHANS), Bengaluru.

\section{The Project}

A case in point is the implementation and evaluation of the NIMHANS-ECHO blended training program in a rural south Indian District of Karnataka state. Project ECHO (Extension for Community Healthcare Outcomes) was originally conceptualized about a decade back to provide best practice care to rural and remote communities which are otherwise underserved. The "Hub" and "Spoke" model links an interprofessional specialist team at an academic centre of expertise (the Hub) with Primary Care Doctors (PCDs) (Spokes) across the states [9-11]. Each ECHO session consists of spoke-led presentations of anonymized patient cases and discussion of the cases by where hub experts provide advice on the management. This is followed by a didactic on health-related topic from the curriculum for the course.

This ongoing project is a collaboration between investigators from NIMHANS, Bengaluru, India (a tertiary care neuropsychiatric academic institute of national importance, Ministry of Health and Family Welfare, Govt. of India), Project ECHO and the Department of Health and Family Welfare Services, Govt. of Karnataka, India. The objectives are to examine the impact of two different types of training methods for non-specialist medical officers and community health workers of Ramanagara District of Karnataka, a state in south India. The control group received the traditional training model, which consists of one-time onsite classroom training and no further mentoring/handholding. The study group receives extended mentoring for six months in addition to the onsite classroom training. A training hub was established inside the premises of the Ramanagara District training Centre (that comes under the administrative control of the District Health authorities), and the mentoring was done remotely using digital technology [2] consisting of live video-based interactions with healthcare workers, with the aim of skill-building. Sessions included case-based discussions and presentations on dedicated topics related to case-finding, simple interventions that community health workers can do, and referral mechanisms. Participants are the Community Health Workers [CHWs: Accredited Social Health Activists (ASHAs) and Auxiliary Nurses Midwives (ANMs)] of the three primary health centres (PHCs) of the study group (total of $37 \mathrm{CHWs}$ ). The CHW were specifically chosen for this study, since they would be the one doing door to door surveys and they would be knowing the villagers even at personal level. The sessions occur fortnightly for each of the three study PHCs, one at a time. Total six sessions are planned for each of the study PHCs covering the following aspects: (1) sad, worried and nervous persons (b) violent, fearful and disorganized persons (3) suicide and how to manage suicidality (4) behavioural problems related to substance use (5) do's and don'ts (and myths) about mental health in the 
community and (6) rehabilitation and disability benefits that are available to those with mental illnesses. Curriculum for the sessions was prepared by the investigators keeping in mind specific expected roles of the CHWs (identification, referral and basic counselling). As per the Project ECHO protocol, each session includes anonymised case presentations by the CHWs (generally related to the topic of the day) followed by discussion. Case discussion is followed by a didactic on the day's topic by the hub experts (in local language, Kannada). Additionally, each session also includes a recap of the fortnight's work and a problem solving discussion related to any work related difficulties that the CHWs may face. CHWs are supposed to log in independently or all gather in their respective PHCs for each of the sessions. To date, we have completed 20 online sessions. Since the ultimate purpose of this implementation research is to develop insights that could be generalized across India, we set up the training hub (Studio) in the district headquarters (Ramanagara) instead of NIMHANS that is located in a metropolitan city. The goal was to further decentralize and empower the health system at the district level, which is essential to integrate mental health into the general health care. The district headquarters hub is involved in training the health care workers of 3 study group PHCs. An important outcome measure of the project is the number of patients (with mental health problems) identified, referred and given interventions. Below we describe the various technology (and related) administrative challenges we encountered in launching and sustaining these online mentoring sessions.

\section{Challenges About Physical Space in Setting Up the Hub Studio}

The initial plan was to set the hub up within the premises of the district hospital. However, due to space constraints, the physical space was provided by the District Training Centre (DTC), Ramanagara, situated about $500 \mathrm{~m}$ away from the District Hospital. Although state-level authorities permitted us to use the physical space for conducting the training, another goahead was required from the principal of DTC as multiple other training activities also occur at the same site. At times, training sessions had to be rescheduled due to calendar conflict. Rescheduling was a complicated task since it required coordinating with a whole group of CHWs to set up a new time for the session. This duplicate work took a lot of time and was sometimes frustrating.

\section{Challenges Related to Internet and Software}

Additionally, due to the lower bandwidth at the district level hub, which is significantly lower than that of the metropolitan city, there were periodic disruptions in internet connectivity. Also, occasional power cuts resulted in delays (and sometimes cancellation of sessions). Lastly, CHWs' areas of operation are in more peripheral locations where internet connectivity is even worse. Therefore all 9 Sessions to date have happened after the CHWs gathered in their respective nodal PHCs. Attending classes online, utilizing smartphones to engage in digital learning requires some technical expertise and experience. CHWs, who are generally 10th class graduates, faced difficulties engaging with the video conferencing software (zoom application in this case) to join the classes, selecting appropriate audio options, and minimizing noise interference during a session. CHW's needed repeated instructions and training regarding using the technology. Some had issues in the comprehension of the English language that 
came in their way of signing and logging in. The Junior Research Fellow had to make dedicated visits to PHCs for familiarizing CHWs with the application. Apart from technical difficulties, administrative issues such as frequent transfer of authorities and controlling officers and a lack of dedicated space for the studio compounded the problem. Lastly, though internet penetration has significantly increased in the country, its actual presence in rural hinterlands is still not optimal [12].

\section{Challenges in Training the CHWs}

Most of the CHWs are lay persons with education less than 10 standard, because of which there were difficulties in explaining the concepts to them. The CHWs also faced resistance while they were doing house to house surveys. The residents of the villages were not very forth coming when the help was offered and showed lot of resistance when they were told their family member had signs suggestive of mental illness. The response from such people has been that they would take the patient to a religious place or to a spiritual healer for the treatment. This reflects that the stigma is still widely prevalent in our country, more so in rural areas as pointed out previous studies [13, 14]. Another important obstacle was that the CHWs were not paid regular salaries by the state government. For majority of the part they were on strike to increase their salary and this further delayed the process of training them. COVID-19 was another hurdle, because of which the CHWs were not welcomed by the villagers, fearing that they would be carriers of infection as travel and meet multiple people on day to day basis. The research being conducted by NIMHANS itself created a hurdle. People in the interior villages felt that NIMHANS is meant for treatment for severely mentally ill and they are not severely mentally ill, so they refused accepting any form of suggestion by our team. This highlights the importance of incorporating psychiatric services in general hospital and if the same research was conducted by general hospital this obstacle might not have come-up.

\section{Opportunities}

Despite the challenges described above, persistent efforts were successful, and the results were gratifying. Slowly, but surely there was a positive change in the confidence and attitude of CHWs towards mental health. They started identifying cases during their routine visits to households. Some of the CHWs were proactive in their initiatives and would accompany patients to their respective PHCs to ensure that they received treatment. When patients could not be treated at PHCs, and required referral to higher centers, the CHWs coordinated the care by interacting with the research team by telephone or video. Psychiatric case-finding by the CHWs is a relatively new concept in the public mental health of India $[15,16]$, and this augurs well for the future. In fact, in 6 months, 37 CHWs (of the study PHC) have identified more than 220 patients with deemed psychiatric problems residing within their communities. It is reasonable to assume that many of them may not have reached treatment centers at all, but for the active outreach and coordination by the CHWs. Technology has played a significant role in this endeavour. It has shown that if there is persistent and committed effort, the challenges (in its use) can be overcome towards bridging the treatment gap. 


\section{Conclusions and Future Directions}

Use of technology in public mental health in India is a relatively new arena that has shown a lot of promise towards bridging the gap. Several initiatives are underway with the potential to exponentially increase the number of trained human resources and expand access for patients. However, for success, persistent efforts are required by committed personnel willing to engage at the grass-root level to overcome technical challenges. To handle poor internet connectivity, one could have an internet "Kiosk" at every village, where high-speed internet can be accessed [17].

\section{The Author Declaration}

1. This paper has not been published or simultaneously submitted, or already accepted for publication elsewhere.

2. The manuscript has been read and approved by all the authors, the requirements for authorship as stated earlier in this document have been met, and each author believes that the manuscript represents honest work. appropraite

3. To the best of our knowledge, this work does not infringe upon any copyright or property right of any third party.

Acknowledgements The researchers would like to thank the patients and their respective families, the health care professionals of the state of Karnataka including the health administrators, DMHP teams, Primary care doctors, Accredited Social Health Activists (ASHA) workers, Auxiliary Nurse Midwives (ANMs) for their contribution in effective Implementation of the trial. This work is supported by the Indian Council Medical Research (ICMR) under Capacity Building Projects for National Mental Health Program, ICMR-NMHP. We thank Dr. Soumya Swaminathan (then Secretary, Dept. of Health Research, DHR), Dr. Balram Bhargav (current Secretary DHR), Prof. V.L. Nimgaonkar, Prof. Smita N. Deshpande, Dr. Ravinder Singh and Dr. Harpreet Singh. We thank the faculty of 'Cross-Fertilized Research Training for New Investigators in India and Egypt' (D43 TW009114, HMSC File No. Indo-Foreign/35/M/2012-NCD-1, funded by Fogarty International Centre, NIH). We are also thankful to National Coordinating Unit of ICMR for NMHP Projects for their constant support and guidance. We thank Data Management Unit of ICMR for designing the database. The content of this manuscript is solely the responsibility of the authors and does not necessarily represent the official views of NIH or ICMR. NIH and ICMR had no role in the study's design and conduct; collection, management, analysis, and interpretation of the data; preparation, review, or approval of the manuscript; and decision to submit the manuscript for publication.

Authors' Contributions CN Kumar, PK Chand, N Manjunatha, SB Math, J Thirthalli, R Parthasarathy, S Reddy and $\mathrm{S}$ Arora were involved in planning this project.

CN Kumar, PK Chand, N Manjunatha, SB Math, J Thirthalli, AA Manjappa and R Parthasarathy, were involved in execution of this project.

BC Malathesh, FA Ibrahim, PL Nirisha, CN Kumar and S Arora were involved in prepapration of the manuscript.

Funding Indian Council of Medical Research vide sanction letter no.5/4-4/151/M/2017/NCD-1.

Data Availability Not applicable.

\section{Compliance with Ethical Standards}

Conflict of Interest/Competing Interests Nil. 
Ethics Approval National Institute of Mental Health and Neuro Sciences, Bengaluru; Letter no: NIMHANS/ EC (BEH.SC.DIV) 6th MEETING/2017.

Consent to Participate Relevant consent was obtained by patients when were enrolled for the above study.

Consent for Publication Obtained.

Ethical Approval National Institute of Mental Health and Neuro Sciences, Bengaluru; Letter no: NIMHANS/ EC (BEH.SC.DIV) 6th MEETING/2017.

Code Availability Not applicable.

\section{References}

1. Singh OP. Closing treatment gap of mental disorders in India: opportunity in new competency-based medical Council of India curriculum. Indian J Psychiatry. 2018;60:375-6. https://doi.org/10.4103/ psychiatry.IndianJPsychiatry_458_18.

2. Mehrotra K, Chand P, Bandawar M, Rao Sagi M, Kaur S, G A, et al. Effectiveness of NIMHANS ECHO blended tele-mentoring model on integrated mental health and addiction for counsellors in rural and underserved districts of Chhattisgarh, India. Asian J Psychiatr. 2018;36:123-7. https://doi.org/10.1016/j. ajp.2018.07.010.

3. Chand P, Murthy P, Gupta V, et al (2014) Technology enhanced learning in addiction mental health: developing a virtual knowledge network: NIMHANS ECHO. In: 2014 IEEE Sixth International Conference on Technology for Education. pp 229-232.

4. Sagi MR, Aurobind G, Chand P, Ashfak A, Karthick C, Kubenthiran N, et al. Innovative telementoring for addiction management for remote primary care physicians: a feasibility study. Indian J Psychiatry. 2018;60: 461-6. https://doi.org/10.4103/psychiatry.IndianJPsychiatry_211_18.

5. Agarwal PP, Manjunatha N, Gowda GS, Girish Kumar MN, Shanthaveeranna N, Kumar CN, et al. Collaborative Tele-neuropsychiatry consultation Services for Patients in central prisons. Journal of Neurosciences in Rural Practice. 2019;10:101-5. https://doi.org/10.4103/jnrp.jnrp_215_18.

6. Khan K, Mathur A, Kaur S, Ganesh A, Chand P. Profile of cases discussed in innovative tele-ECHO mentoring program on addiction management. Asian J Psychiatr. 2020;52:102060. https://doi.org/10.1016/ j.ajp.2020.102060.

7. Manjunatha N, Kumar C, Math S, Thirthalli J. Designing and implementing an innovative digitally driven primary care psychiatry program in India. Indian J Psychiatry. 2018;60:236-44. https://doi.org/10.4103/ psychiatry.IndianJPsychiatry_214_18.

8. Pahuja E, Santosh K, Harshitha N, Fareeduzaffer, Manjunatha N, Gupta R, et al. Diploma in primary care psychiatry: an innovative digitally driven course for primary care doctors to integrate psychiatry in their general practice. Asian J Psychiatr. 2020;52:102129. https://doi.org/10.1016/j.ajp.2020.102129.

9. Arora S, Kalishman S, Thornton K, Dion D, Murata G, Deming P, et al. Expanding access to hepatitis C virus treatment-extension for community healthcare outcomes (ECHO) project: disruptive innovation in specialty care. Hepatology. 2010;52:1124-33. https://doi.org/10.1002/hep.23802.

10. Arora S, Thornton K, Komaromy M, Kalishman S, Katzman J, Duhigg D. Demonopolizing Medical Knowledge. Demonopolizing Medical Knowledge: Academic Medicine. 2014;89:30-2. https://doi.org/10. 1097/ACM.0000000000000051.

11. Arora S, Kalishman S, Dion D, Som D, Thornton K, Bankhurst A, et al. Partnering urban academic medical centers and rural primary care clinicians to provide complex chronic disease care. Health Aff. 2011;30: 1176-84. https://doi.org/10.1377/hlthaff.2011.0278.

12. Biswas $\mathrm{S}$ (2019) India's digital sprint is leaving millions behind. BBC News.

13. Grover S, Shouan A, Sahoo S. Labels used for persons with severe mental illness and their stigma experience in North India. Asian J Psychiatr. 2020;48:101909. https://doi.org/10.1016/j.ajp.2019.101909.

14. Kudva KG, El Hayek S, Gupta AK, et al. Stigma in mental illness: perspective from eight Asian nations. Asia Pac Psychiatry. 2020;12:e12380. https://doi.org/10.1111/appy.12380.

15. Chatterjee S, Naik S, John S, Dabholkar H, Balaji M, Koschorke M, et al. Effectiveness of a communitybased intervention for people with schizophrenia and their caregivers in India (COPSI): a randomised controlled trial. Lancet. 2014;383:1385-94. https://doi.org/10.1016/S0140-6736(13)62629-X. 
16. Pradeep J, Isaacs A, Shanbag D, et al. Enhanced care by community health workers in improving treatment adherence to antidepressant medication in rural women with major depression. Indian J Med Res. 2014;139:236.

17. Digital Villages. In: VNL. http://www.vnl.in/rural-uso-funds-solutions/digital-villages. Accessed 10 Aug 2020.

Publisher's Note Springer Nature remains neutral with regard to jurisdictional claims in published maps and

institutional affiliations.

Dr. Barikar C. Malathesh is an Assistant Professor of Psychiatry at NIMHANS, Banglore, India. He further obtained fellowship in Forensic Psychiatry from National Institute of Mental Health and Neurosciences, Bengaluru. He has total of 6 years' experience in the field of psychiatry. Currently working at National Institute of Mental Health and Neurosciences. His areas of interest include Forensic Psychiatry and General Adult Psychiatry.

Dr. Ferose Azeez Ibrahim , currently works as a Senior Resident in the Department of Psychiatry at the National Institute of Mental Health and Neurosciences (NIMHANS). His areas of interests are Community Psychiatry, Addiction Medicine and Forensic Psychiatry. He is also involved in training primary care doctors in the State of Chhattisgarh, India in primary care psychiatry through Telemedicine.

Dr. Lakshmi Nirisha P , MBBS MD (Psychiatry), currently holds post of Assistant professor, in telemedicine Centre, Department of Psychiatry NIMHANS, Bangalore, is involved in training of Non-psychiatry Specialist Medical officers in primary care psychiatry. Her key areas of clinical and research interests include Telepsychiatry, Consultation Liaison psychiatry and geriatric psychiatry.

Dr. Channaveerachari Naveen Kumar, currently hold the position of Professor in the Department of Psychiatry at the National Institute of Mental Health and Neurosciences (NIMHANS). I am also the Consultant of Community Mental Health Unit, Forensic Psychiatry services, Non-Invasive Brain Stimulation Team and Telemedicine Services at NIMHANS. I have been running community intervention programs in two rural taluks of Karnataka since the past decade: Thirthahalli and Turuvekere. My areas of interests are Electroconvulsive therapy, Forensic Psychiatry, Psychiatric Rehabilitation, and community psychiatry.

Dr. Prabhat Kumar Chand holds the position of Additional Professor, Department of Psychiatry at National Institute of Mental Health and Neurosciences (NIMHANS). He is also the Co-ordinator of the Virtual Knowledge Network NIMHANS ECHO. His research specializations are Public Mental Health, Substance use disorder, Comorbidity, Use of Technology for tele-mentoring and training, linking academic centres to community for better health care.

Dr. Narayana Manjunatha is currently an Associate Professor in the Department of Psychiatry at National Institute of Mental Health and Neurosciences (NIMHANS). He has been involved in the NMHP activities over the past 7 years and his fields of interests are Primary Care Psychiatry, Community Psychiatry and Telemedicine.

Dr. Suresh Bada Math is a Professor in the Department of Psychiatry at National Institute of Mental Health and Neurosciences (NIMHANS). He is the In-charge Head of Community Psychiatry and Telemedicine Centre. He is also a consultant of Forensic Psychiatry. His areas of interests include Forensic Psychiatry, Community Psychiatry, Telemedicine, Human Rights of the persons with mental illness and Obsessive-Compulsive Disorder.

Dr. Jagadisha Thirthalli is a Professor in the Department of Psychiatry at National Institute of Mental Health and Neurosciences (NIMHANS). He is the In-charge Head of the Psychiatric Rehabilitation Services at NIMHANS and his areas of interests are Electroconvulsive Therapy and Psychiatric Rehabilitation.

Dr. Adarsha Alur Manjappa es a psychiatrist working for the District Mental Health Program of Ramanagara District of Karnataka, India for 4 years and he leads the team spearheading the following initiatives (1) provision of clinical services at multiple outpatient clinics spread throughout the district (2) capacity building in mental health throughout the district for various stakeholders including non-specialist medical officers, nurses, community health 
workers, pharmacists and many others (3) Information Education and Communication Activities involving schools, colleges, police, members of local self-governance mechanisms, elected representatives, judicial officers etc.

Dr. Rajani Parthasarathy is the Deputy Director, Mental Health in the Department of Health and Family Welfare, Government of Karnataka. She is the nodal person for mental health in Karnataka at the level of Directorate for Health Services.

Dr. Shanivaram Reddy is an Assistant Professor in the Department of Psychiatric Social Work at NIMHANS, Bengaluru. His fields of interests are Psychiatric Rehabilitation, Psychiatric disability, caregiver's education and support programs, Community based rehabilitation \& advocacy for mentally ill.

Dr. Sanjeev Arora is the Director and Founder of Project Echo. He is a Distinguished and Regents' Professor of Medicine with tenure in the Department of Internal Medicine at the University of New Mexico Health Sciences Center (UNMHSC). Dr. Arora developed the ECHO model and founded Project ECHO (Extension for Community Healthcare Outcomes) to dramatically improve both capacity and access to specialty care for rural and underserved populations. 